\title{
Carotid Plaque Hemodynamics
}

\author{
Andreas Harloff \\ Department of Neurology, University Hospital Freiburg, Freiburg, Germany
}

\section{Key Words}

Vulnerable plaque $\cdot$ Rupture $\cdot$ Carotid artery $\cdot$ Wall shear stress $\cdot$ Tensile stress

\begin{abstract}
Internal carotid artery (ICA) plaques constitute one major source of retinal and cerebral brain embolism. Current guidelines recommend optimal treatment of cardiovascular risk factors and recanalization based on the degree of ICA stenosis. However, ICA plaque composition, motion, vascularization, and local hemodynamics have only received limited attention as potential and independent risk factors for plaque rupture. The European Carotid Surgery Trial (ECST) showed an increased risk of stroke recurrence even in moderate stenosis if the plaque surface was ulcerated in angiography. Further indicators of plaque instability and thus vulnerability were established by native or contrast-enhanced two-dimensional duplex ultrasound, CT, and MRI. Due to high soft tissue contrast, multi-contrast MRI seems to be ideally suited to identify plaque compositions that are prone to rupture, although data from large clinical trials proving the independent predictive value of plaque morphology are lacking. The role of cardiovascular risk factors for atherosclerosis of the common carotid artery is well established. Nevertheless, little is known concerning the impact of local hemodynamics on plaque development, progression, and rupture. Wall shear stress, the friction force acting on the endothelium of the vessel wall, was shown to be able to induce local atherosclerosis and vulnerable plaques in animal models. Plaque movement and deformation was limited to investigations using ultrasound in order to identify plaques at risk. Similarly, models to calculate tensile plaque stress seem to be able to identify peak mechanical stress acting on plaque surfaces that make such regions susceptible to rupture. In this review, current evidence regarding the correlation of plaque location, composition, and local hemodynamics at the carotid artery bifurcation will be presented. Moreover, the potential benefit of a future comprehensive and individual risk assessment will be discussed.
\end{abstract}




\section{Introduction}

Internal carotid artery (ICA) stenosis constitutes one major source of ipsilateral retinal and cerebral embolism. Current guidelines recommend optimal treatment of cardiovascular risk factors in patients with carotid atherosclerosis. Recanalization of ICA stenosis is primarily indicated if the degree of stenosis is $\geq 50 \%$ following North American Symptomatic Carotid Endarterectomy Trial (NASCET) criteria [1] and in particular if that stenosis was symptomatic. However, a number of patients with 70\% ICA stenosis remain asymptomatic for years whereas others with only $30 \%$ ICA stenosis suffer ipsilateral stroke of otherwise cryptogenic etiology. Therefore, the degree of stenosis does not provide complete information and it is obvious that other factors must contribute to plaque stabilization or rupture. The histological composition of plaques is a promising candidate: the existence of a stable plaque in one patient and of a vulnerable plaque type in another patient is probably significant regarding the hazardousness of ICA plaques.

For clinicians it is essential to identify factors that promote progression of atherosclerosis and that ultimately predict plaque rupture. Moreover, in patients developing, for example, $70 \%$ ICA stenosis despite intensive conservative treatment, recanalization therapies should be directed in time and before stroke occurs. Potentially, this can be achieved by characterization of plaque components such as plaque hemorrhage or a large lipid-necrotic core. These features can be demonstrated using modern imaging techniques such as high-resolution ultrasound, MRI, and CT in combination with PET imaging [2]. Additionally, including analysis of individual blood flow at the site of such plaque seems to be necessary to comprehensively evaluate all factors that influence plaque destabilization and in the end retinal or cerebral embolism. Mechanical forces such as wall shear stress (WSS) [3] or tensile stress [4] that act on the vessel endothelium or on the plaque volume could be ancillary to stratify the individual risk of atherogenesis and plaque rupture.

A more comprehensive risk assessment of predictors of plaque rupture could finally lead to an optimal treatment providing maximum security and minimal side effects for the individual. Therefore, it is the aim of this review to give an overview about current concepts and evidence concerning vulnerable plaques, with particular emphasis on plaque hemodynamics.

\section{Plaque Morphology}

\section{The Concept of Vulnerable Plaques}

The NASCET [5], European Carotid Surgery Trial (ECST) [6] and Asymptomatic Carotid Surgery Trial (ACST) [7] investigated the benefit of carotid endarterectomy and of surgery compared to conservative treatment, respectively. However, they mainly focused on the degree of ICA stenosis. Nonetheless, a subgroup analysis of the ECST trial revealed that the risk of stroke was higher in patients with additional plaque ulcerations in digital subtraction angiography and indicated that factors besides lumen narrowing play a role in embolism.

The term vulnerable plaque was introduced by Muller et al. [8] to describe atherosclerotic plaques that are prone to rupture and the underlying cause of most clinical coronary events. Subsequently, this concept was transferred to carotid artery plaques and compared with specific features in histopathology. A consensus paper for the definition of vulnerable plaques was provided in 2003 in order to 'identify apparently healthy subjects at risk of future events' by proposed plaque criteria [9]. It consisted of the following major criteria of plaque vulnerability: active inflammation, thin cap with a large lipid core, endothelial denudation with superficial platelet aggregation, a fissured plaque or stenosis $>90 \%$. Additionally, minor 
criteria were, for example, intraplaque hemorrhage or positive outward remodeling. However, definitive proof of the vulnerable plaque concept by an appropriate animal model of atherosclerosis or by human studies providing a clear cause-and-effect relationship does not yet exist [10]. Therefore, the diagnostic and predictive accuracy of imaging techniques of vulnerable plaques still need to be evaluated in large clinical trials [11].

\section{Imaging of Vulnerable Plaques}

A number of noninvasive diagnostics including ultrasound, CT, MRI, and PET exist to date and allow characterization of plaque composition. However, the information concerning the detailed tissue components and accuracy for the determination of vulnerable plaques is variable [2]. In this part of the review, current ultrasound techniques and MRI approaches will be highlighted. Both modalities do not expose patients to radiation in contrast to CT or PET in combination with CT or MRI [2]. Furthermore, ultrasound and MRI provide detailed plaque information with high spatial resolution and, in the case of MRI, high soft tissue contrast. Therefore, they appear as the most promising tools for future diagnostic or monitoring of patients with ICA stenosis.

\section{Ultrasound}

Plaque Echogenicity and Microemboli

An indirect stratification of individual plaque risk and thus vulnerability was performed in the Asymptomatic Carotid Emboli Study (ACES) trial. Patients with previously asymptomatic and $\geq 70 \%$ ICA stenosis were monitored for cerebral microemboli of the middle cerebral artery in Doppler ultrasound. Subjects with a high rate of microemboli had a significantly higher risk of transient ischemic attack during follow-up compared to those with only few microembolic signals [12]. In a subgroup analysis, echolucency of asymptomatic $\geq 70 \%$ ICA stenosis in longitudinal and transverse B-mode ultrasound was assessed on a five-step visual grading scale. Patients with echolucent plaques had a hazard ratio (HR) of $\sim 6$ for ipsilateral stroke, and in combination with the positive microemboli signals at baseline HR the ratio increased to $\sim 10$. These findings demonstrate the potential of comparable and readily available ultrasound techniques to identify initially asymptomatic patients with obvious vulnerable ICA plaques [13].

\section{Plaque Surface Motion}

Another interesting application of ultrasound is the monitoring of plaque surface motion over time. Three-dimensional (3D) data of plaque motion and surface deformation were acquired in the study of Meairs et al. [14] using 4D B-mode ultrasound providing a spatial and temporal resolution of $\sim 0.2 \mathrm{~mm}^{3}$ and 25 frames/s, respectively. Data were analyzed by motion detection algorithms, and apparent velocity fields (i.e. optical flow) were calculated. These were visualized as vectors representing movement of the plaque surface between initial 3D coordinates of the plaque volume and subsequent coordinates during the heart cycle. The authors hypothesized that multiple tears within symptomatic plaques would make plaques prone to deformations caused by the mechanical forces of blood flow. Thus, an increased plaque motion would be detectable. Accordingly, stable plaques should not show deformations and changes in the plaque surface. Atheroma of 22 symptomatic and 23 asymptomatic patients with 50-90\% ICA stenosis (NASCET criteria) were examined and compared. Drawbacks of the technique included extensive plaque calcifications or high-grade ICA stenosis hampering analysis in one third of the patients. The maximal discrepant surface velocity in systole (i.e. the maximum of the differences between maximal and minimal 


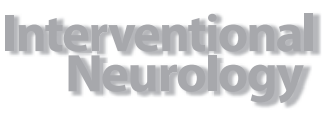

\begin{tabular}{l|l}
\hline Intervent Neurol 2012;1:44-54 \\
\hline DOI: 10.1159/000338360 & $\begin{array}{l}\text { C 2012 S. Karger AG, Basel } \\
\text { www.karger.com/ine }\end{array}$ \\
\hline Published online: April 25, 2012
\end{tabular}
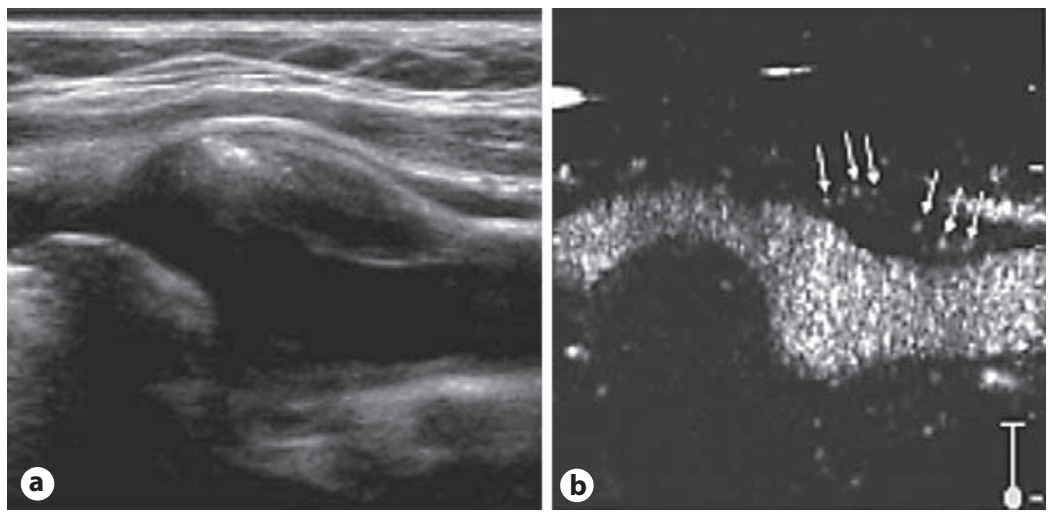

Fig. 1. Carotid artery plaque with intraplaque neovascularization on CEUS. a Longitudinal B-mode image of an ICA plaque of predominant hypoechogenicity with a circumscript hyperechogenic calcification at the left border of the atheroma. b A spot-like increase in echogenicity within the plaque becomes visible due to transition of the vasa vasorum by the microbubbles of the contrast agent (arrows). Reproduced as a modified figure with permission from Staub et al. [18].

surface velocities of successive 3D frame volumes) was significantly higher in symptomatic $(3.9 \mathrm{~mm} / \mathrm{s})$ versus asymptomatic $(0.6 \mathrm{~mm} / \mathrm{s})$ plaques, indicating inherent plaque movement in symptomatic patients. These findings were confirmed in another study in healthy volunteers and in case reports using only 2D ultrasound [15-17]. However, the technique is complex and requires both high-resolution $3 \mathrm{D}$ ultrasound and dedicated software to calculate and quantify plaque motion.

\section{Contrast-Enhanced Ultrasound}

Vasa vasorum (VV) represent a network of small vessels that supply and drain the walls of larger arteries or veins. Early vessel inflammation/atherosclerosis seems to lead to hyperplasia of the adventitial VV whereas in advanced atherosclerosis new microvessels develop in the intima and media layer and thus in the plaque itself. Such changes were found in animal models, autopsy studies, and clinical reports and indicate that an increase in new microvessels also increases the development of rupture-prone atheroma. Interestingly, intraplaque hemorrhage, which has gained considerable significance as a parameter of vulnerable plaques, seems to result from the development of leaky neointimal vessels [18].

Contrast-enhanced ultrasound (CEUS) is an easy, accessible, and promising imaging tool that can be used to noninvasively visualize $\mathrm{VV}$ of the adventitia and of carotid artery plaques. Ideally, a B-mode image of the plaque is taken before CEUS in order to discriminate between areas of the plaque that are echolucent due to calcification or due to subsequent contrast enhancement. One of the many available contrast agents is injected into a peripheral vein as a bolus followed by saline injection. Then, high-resolution ultrasound (7- to $10-\mathrm{MHz}$ ultrasound probe) is applied to the carotid wall and plaques and can be used in combination with contrast imaging software providing pulse inversion or harmonic imaging. Contrast agents consist of gas-filled microbubbles with a diameter of 1-4 $\mu \mathrm{m}$ which allows them to pass microvessels. These very small bubbles oscillate or burst as soon as they are insonated by ultrasound, enhance the reflection of ultrasound waves, and consecutively increase echogenicity in ultrasound images [19].

The mechanical index should be lowered to $<0.2$, resulting in an echolucent or hypoechoic signal of the adventitia and of the plaque. Accordingly, an increase in echogenicity in the wall or in the plaque itself after contrast injection can be identified as contrast en- 
hancement due to tissue perfusion (fig. 1). In addition to imaging of the VV, CEUS provides an improved delineation of plaque surfaces and identification of plaque irregularities or ulcerations. Moreover, in some patients CEUS can be beneficial to discriminate near-occlusion from occlusion of the distal ICA due to the improved visualization of the residual lumen and minimum blood flow.

However, a standardized quantification of the extent of the VV has to be established. In complement to measured semiquantitative two- or four-step scores, changes of signal within the plaque compared to the signal of blood were used to quantify plaque or adventitial hypervascularization. In conclusion, data from animal models and human studies show that CEUS is able to measure the presence and the extent of the VV network and of plaque neovascularization.

\section{Magnetic Resonance Imaging}

Quantification of the VV can also be identified by MRI using dynamic contrast material-enhanced MRI as recently demonstrated in 28 patients with carotid or coronary artery disease [20]. The strength of current MRI techniques, however, is the ability to identify key determinants of plaques using multi-contrast imaging. To date, measurement accuracy and robustness and the reliable identification of different plaque constituents has been proven by several groups [2]. Different plaque components, in particular the lipid-rich necrotic core, plaque hemorrhage, and the fibrous cap thickness measurements or rupture detection can be accurately identified due to their typical appearance and intensities in different MR sequences [21]. Multi-contrast MRI protocols typically consist of time-of-flight angiography, T1, T2, and proton density weighted sequences. Some authors included T1 postcontrast application for the identification of an inflammation of the fibrous cap or for the discrimination of calcification within carotid or aortic atheroma [21,22].

Saam et al. [23] examined the carotid bifurcations of 192 subjects that were recruited from the vascular ultrasound laboratory using multi-contrast MRI at $1.5 \mathrm{~T}$ and evaluated the existence of vulnerable [i.e. American Heart Association (AHA) classification type VI] plaques. The incidence of such plaques was $\sim 75 \%$ in $80-99 \%$ ICA stenosis (NASCET criteria) and decreased with decreasing lumen narrowing. Interestingly, type VI plaques were found in $\sim 22 \%$ of the patients with only low to moderate stenosis $(16-49 \%)$ that would usually not undergo recanalization following current guidelines. These findings show that MRI is able to detect patients with an obviously low risk if the degree of stenosis is considered but potentially can detect those with a high risk if plaque composition is regarded. The incidence of complicated ICA plaques on the side of brain infarction compared to the asymptomatic contralateral side is currently being investigated in a multi-center trial (CAPIAS - carotid plaque imaging in acute stroke).

\section{Summary}

The ultrasound and MRI techniques presented here seem highly promising for the discrimination of carotid plaques at risk. However, further evaluation in future clinical trials is necessary for both techniques: in ultrasound the value of microemboli detection in asymptomatic ICA stenosis, as well as of plaque surface or plaque volume motion, and the quantification of VV, and in MRI, the detection of vulnerable plaques by current and future multicontrast MRI protocols. The trials have to prove that it is beneficial to identify patients at risk based on both stenosis degree and stenosis morphology. Finally, the fastest and most accurate technique of these approaches that is applicable in clinical practice needs to be identified. These methods are of interest for patients with $\geq 50 \%$ ICA stenosis but especially for those with ICA stenosis of only 30-50\% that currently do not undergo recanalization. 


\section{Plaque Hemodynamics}

\section{Specifics of the Carotid Bifurcation}

The carotid bifurcation guides blood from the common carotid artery (CCA) to the brain supplying ICA, and to the external carotid artery (ECA) that supplies the skin and muscles of the head. Accordingly, there is flow separation together with dilatation of the distal CCA/proximal ICA, causing a deceleration of blood flow and thus generating a typical helical flow pattern within the ICA. Such hemodynamics have been shown in flow and computational fluid dynamics (CFD) models and can now be measured in vivo in humans and visualized in 3D using flow-sensitive 4D MRI [24]. The characteristic hemodynamic situation at the proximal ICA bulb is coincident with the predilection site for the occurrence of ICA plaques and stenosis.

However, this anatomic feature is necessary both for the distribution of blood flow and for the accurate measurement of local blood pressure by baroreceptors that are located at the border zone of the media and adventitia of the wall of the ICA bulb. These quantify local pressure or pressure changes and send signals to the medulla oblongata of the brain which regulates arterial blood pressure by inhibition of the sympathetic and stimulation of the parasympathetic nervous system.

\section{Carotid Plaque Hemodynamics}

The role of cardiovascular risk factors for the development and progression of generalized atherosclerosis is well established. In contrast, the role of local hemodynamics such as WSS or tensile stress in plaque development, progression, and rupture in humans is only partially understood. Initially, early atherosclerosis and low grade ICA stenosis caused by an atheroma is compensated by outward remodeling to a certain extent, leading to vessel dilation to minimize the stenotic effect. These early changes are most likely due to low and oscillatory shear stress. Further plaque progression constitutes an increasing obstruction of blood flow that needs to traverse the residual vessel lumen. The consecutive interaction of mechanical forces on the upstream plaque shoulder enhances the pressure on the proximal plaque area and can be measured as tensile stress. One can easily imagine that consecutive and rapid changes of blood pressure or changes in plaque configuration, i.e. a steep instead of streamlined inlet, increase stress on the plaque and lead to plaque rupture depending on plaque stability.

\section{Wall Shear Stress}

Given laminar blood flow conditions the lowest velocities are found at the vessel wall, whereas maximum velocities occur in the lumen center and form a nearly parabolic flow profile. The wall shear rate is the ratio between adjacent blood flow velocities close to the vessel wall. Accounting for blood viscosity allows the calculation of WSS in newtons per square meter. WSS represents the friction force of flowing blood that acts on the endothelial wall. WSS can vary considerably throughout vascular beds and is especially altered at the outlet or at the inner curvature of arteries. Low WSS and oscillating WSS, i.e. a local and rapid change in flow direction within the cardiac cycle, are considered to be responsible for the development and progression of local atherosclerosis. These flow disturbances promote sustained activation of a number of atherogenic genes in the endothelial cells such as monocyte chemotactic protein- 1 that induces monocyte infiltration into the arterial wall, and platelet-derived growth factors that enhance the turnover of endothelial cells and migration of smooth muscle cells into the subintimal space $[25,26]$. A precise calculation of absolute WSS requires a method capable of high spatial and temporal resolutions. In addition, blood flow and accordingly WSS are vector quantities and therefore optimal diagnostics of WSS need to be able to quantify blood flow in 3D. 
In an experiment of cultured bovine endothelial cells [25], different patterns of cellular organization dependent on the type of flow, i.e. no flow, laminar flow, or oscillating flow, were shown. Laminar flow induced a homogeneous orientation of cells along the flow direction and thus a compact organization with only minimal intercellular gaps. By contrast, oscillating flow led to a copper stone pattern suggesting a more vulnerable cellular network. In an animal model of Apo E knockout mice that were fed a Western diet, Cheng et al. [27] followed the spontaneous development of early atherosclerotic lesions in the aortic arch and the supra-aortic arteries over a period of 6 weeks. Furthermore, a cast positioned at one ICA caused $\sim 70 \%$ stenosis. As expected, histopathology revealed that atherosclerotic lesions occurred at the outlet of the supra-aortic arteries and at the inner curve of the aortic arch. More interestingly, however, zones of increased WSS such as at the stenotic ICA segment were protected from atherosclerosis, but regions of high oscillatory shear stress (distal to the stenosis) caused stable plaques, and low WSS (proximal to the stenosis) caused vulnerable lesions. Using CFD, Lee et al. [28] studied the distribution of WSS of 64 bifurcations of healthy volunteers in combination with 3D anatomical information provided by MR angiography. Disturbed blood flow was quantified as the surface area exposed to low and oscillatory shear beyond objectively defined thresholds. Moreover, carotid bifurcation geometry was analyzed individually and correlated with the distribution of WSS. As a result, multiple regressions revealed that there was a significant relationship between disturbed flow and both the proximal area ratio, i.e. the ratio between the size of the area of the proximal ICA and ECA compared to the CCA, and bifurcation tortuosity. Accordingly, a large ICA bulb and an elongation of the ICA predisposed to the occurrence of disturbed flow.

However, data from in vivo studies are sparse. Therefore, flow-sensitive 4D MRI was applied to measure WSS distribution along the carotid bifurcation in 32 healthy volunteers and patients with high-grade ICA stenosis before and after carotid endarterectomy at $3 \mathrm{~T}$ [29]. Absolute and oscillating WSS were calculated within standardized analysis planes along the CCA and ICA and using in-house software. Geometrical parameters similar to those presented in the CFD study by Lee et al. [28] were measured in vivo (fig. 2). The temporal and spatial resolution of that method and thus the accuracy of absolute values of WSS were limited compared to CFD studies. However, this study allowed reliable assessment of relative changes in WSS along the carotid bifurcations in a large number of carotid arteries in vivo. The values of oscillatory and low WSS of all healthy volunteers were pooled and the highest and lowest 10 and $20 \%$ of the values of absolute WSS and OSI, respectively, were defined as critical WSS. Interestingly, such WSS was consistently concentrated in proximal bulb regions of the CCA and ICA and thus at the site where carotid artery plaques typically develop. Multiple linear regressions revealed that, similar to the results of Lee et al. [28], critically low absolute WSS significantly correlated with the ICA/CCA diameter ratio. Regions exposed to critically high OSI were significantly correlated with a large bifurcation angle, distinct tortuosity of the ICA, and a large ICA/CCA diameter ratio. Moderate ICA stenosis (i.e. $20-40 \%$ NASCET criteria), however, altered the physiological WSS distribution in the carotid bifurcation due to removal of the ICA bulb, flow acceleration within the stenosis, and poststenotic complex flow patterns. Patients with previously $\geq 50 \%$ ICA stenosis (NACSET criteria) who underwent carotid surgery showed a recovery of flow patterns and distribution of WSS that were comparable to healthy volunteers. Thus, flow-sensitive 4D MRI seems very promising to study the influence of individual bifurcation geometry, plaque formation, and progression on local hemodynamics. Moreover, this technique seems ideally suited for combination with multi-contrast MRI for carotid plaque imaging in order to study fluid-structure interactions in vivo. Yang et al. [30] studied the progression of carotid plaques in 14 patients over 18 months using repeated in vivo multi-contrast MRI at $1.5 \mathrm{~T}$. Plaque wall stress and flow shear stress were calculated using 3D fluid-structure interaction models and matched 


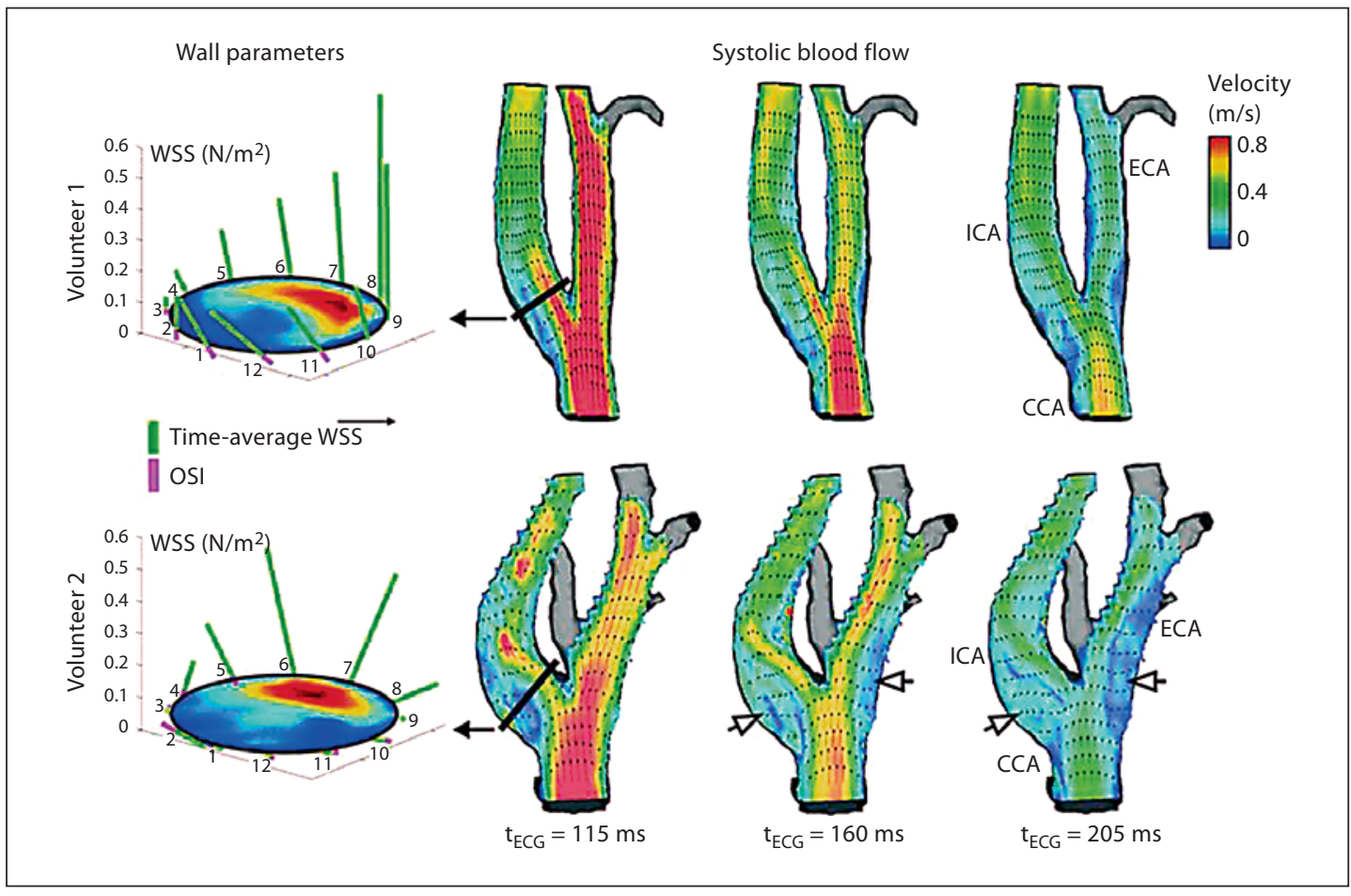

Fig. 2. Quantification and visualization of WSS and blood flow velocities depending on stenosis geometry. Blood flow in two healthy volunteers is visualized using a longitudinal plane transecting the 3D geometry of the carotid bifurcation. The vector fields and color coding of velocity magnitude demonstrate the effect of carotid bifurcation geometry on blood flow patterns and resulting wall parameters (analysis planes for WSS calculation, left) in the proximal ICA. The increased ratio of the ICA/CCA diameter and the larger bifurcation angle in volunteer 2 leads to more complex flow channels and larger areas with low and circulating flow (open arrows). Thus, larger zones of the proximal ICA and ECA are exposed to low WSS and suggest a potentially higher risk of atherogenesis in volunteer 2 compared to volunteer 1 . The geometry parameters measured in volunteer $2 /$ volunteer 1 were: bifurcation angle, $41^{\circ} / 23^{\circ}$; tortuosity, $1.03 / 1.02$, and ICA/CCA diameter ratio, 1.2/1.1, respectively. Reproduced with permission from Markl et al. [29].

with wall thickness increases during follow-up. In the majority of patients, flow shear stress was positively correlated with an increase in wall thickness and indicated an interaction of flow parameters and plaque development.

\section{Tensile Stress}

Tensile stress (measured in $\mathrm{kPa}$ ) is the stress state leading to expansion of a material, i.e. the material length increases in the tensile direction. Accordingly, tensile stress is the opposite of compressive stress. Fracture stress has been defined as tensile stress that is above the required strength of a material to maintain structure (e.g. a plaque tissue) and thus leads to rupture of the plaque. Accordingly, the risk of plaque rupture is high in coincidence with high tensile stress and a vulnerable plaque composed of a thin fibrous cap, a large lipid-rich/necrotic core, and limited stabilizing tissue such as smooth muscle cells or fibrous tissue. The maximum tensile stress can be found at the fibrous cap of the upstream shoulder of the plaque and corresponds with the observation of fissures and ruptures. A high pressure drop over the stenotic segment, due to a high-grade luminal narrowing or a steep inlet of the stenosis, constitutes a predisposition to plaque rupture. Due to much higher values of tensile stress compared to WSS, it appears that tensile stress is the major hemodynamic factor responsible for 
plaque rupture. Calculations of tensile stress are currently performed using CFD models illustrating that even relatively small pressure drops can induce high tensile stress especially at the thinnest site of the fibrous cap [31]. Using in vivo multi-contrast MRI in combination with a $3 \mathrm{D}$ reconstruction of plaque geometry and fluid structure interaction simulation for biomechanical analysis, Gao et al. [32] studied 12 symptomatic patients with previous acute transient ischemic retinal or cerebral ischemia and 8 asymptomatic patients from a specialist neurovascular clinic. In this study, extreme tensile stress was found at the fibrous cap of the proximal plaque shoulder and the local maximum stress levels were significantly higher in symptomatic compared to asymptomatic patients. In addition, an increase in the degree of ICA stenosis correlated with an increase in extreme stress upstream from the plaque throat.

\section{Conclusion}

Future investigations should establish an in vivo diagnostic that provides a fast $3 \mathrm{D}$ assessment of WSS with higher spatial and temporal resolution compared to current protocols in order to monitor early changes in atherosclerosis due to local hemodynamics. Identification of plaques that are prone to rupture could be achieved by calculations of tensile stress. However, this technique is even more time consuming than determination of WSS and currently requires CFD models. The value of predicting plaque development and rupture due to hemodynamics should be determined in longitudinal clinical studies. For the moment, these techniques are of high scientific value, but significant simplifications and acceleration of data analysis are needed before they will be accepted by clinicians and executed in clinical routine.

\section{Clinical Perspective}

In this review, current evidence regarding the correlation of plaque location, composition, and local hemodynamics at the carotid artery bifurcation was described with emphasis on imaging of vulnerable plaques, including the hemodynamic impact of plaque microcirculation and WSS. CEUS and multi-contrast MRI for the detection of vulnerable plaques seem to be especially promising. Standardization of protocols and of quantification is still necessary, though applications in clinical routine appear feasible in the near future provided that large clinical trials have proven their independent value for risk stratification beyond assessment of the degree of ICA stenosis. It seems obvious that WSS is more significant for the initial development of atherosclerosis and for the composition of plaques. The individual anatomy of the carotid bifurcation appears to influence the distribution of WSS, and thus certain individuals might be at an additional risk of atherosclerosis independent of established cardiovascular risk factors. These patients could benefit from an intensified treatment of cardiovascular risk factors. Tensile stress, however, seems to be relevant in later stages of atherosclerosis and the calculation of tensile stress could identify relevant triggers such as an increase in hypertension for immediate plaque rupture.

Further improvement and acceleration of the measurement and analysis of plaque morphology and hemodynamics derived from either ultrasound or MRI could provide a comprehensive and patient-tailored risk assessment. This may improve decision making to select the best treatment (e.g. conservative, stent angioplasty, or surgery). For radiologists or vascular surgeons, assessment of 3D hemodynamics at the carotid bifurcation and simulations of the influence of geometrical changes on blood flow could help to optimize stent positioning and design or to choose eversion endarterectomy instead of patch repair in order to minimize restenosis rates. 


\section{Disclosure Statement}

I declare that I have no conflicts of interest regarding sponsorship or funding arrangements related to my research or related to this article to disclose.

\section{References}

1 von Reutern GM, Goertler MW, Bornstein NM, Sette MD, Evans DH, Hetzel A, Kaps M, Perren F, Razumovky A, von Reutern M, Shiogai T, Titianova E, Traubner P, Venketasubramanian N, Wong LK, Yasaka M, Neurosonology Research Group of the World Federation of Neurology: Grading carotid stenosis using ultrasonic methods. Stroke 2012; 43:916-921.

2 U-King-Im JM, Young V, Gillard JH: Carotid-artery imaging in the diagnosis and management of patients at risk of stroke. Lancet Neurol 2009;8:569-580.

3 Malek AM, Alper SL, Izumo S: Hemodynamic shear stress and its role in atherosclerosis. JAMA 1999;282:2035-2042.

4 Tang D, Yang C, Zheng J, Woodard PK, Saffitz JE, Sicard GA, Pilgram TK, Yuan C: Quantifying effects of plaque structure and material properties on stress distributions in human atherosclerotic plaques using 3D FSI models. J Biomech Eng 2005;127:1185-1194.

5 Beneficial effect of carotid endarterectomy in symptomatic patients with high-grade carotid stenosis. N Engl J Med 1991;325:445-453.

6 MRC European Carotid Surgery Trial: interim results for symptomatic patients with severe (70-99\%) or with mild (0-29\%) carotid stenosis - European Carotid Surgery Trialists' Collaborative Group. Lancet 1991;337:1235-1243.

7 Halliday A, Mansfield A, Marro J, Peto C, Peto R, Potter J, Thomas D, MRC Asymptomatic Carotid Surgery Trial (ACST) Collaborative Group: Prevention of disabling and fatal strokes by successful carotid endarterectomy in patients without recent neurological symptoms: randomised controlled trial. Lancet 2004;363:1491-1502.

8 Muller JE, Abela GS, Nesto RW, Tofler GH: Triggers, acute risk factors and vulnerable plaques: the lexicon of a new frontier. J Am Coll Cardiol 1994;23:809-813.

-9 Madjid M, Zarrabi A, Burke A, Yuan C, Fitzgerald PJ, Siscovick DS, de Korte CL, Aikawa M, Juhani Airaksinen KE, Assmann G, Becker CR, Chesebro JH, Farb A, Galis ZS, Jackson C, Jang IK, Koenig W, Lodder RA, March K, Demirovic J, Navab M, Priori SG, Rekhter MD, Bahr R, Grundy SM, Mehran R, Colombo A, Boerwinkle E, Ballantyne C, Insull W Jr, Schwartz RS, Vogel R, Serruys PW, Hansson GK, Faxon DP, Kaul S, Drexler H, Greenland P, Muller JE, Virmani R, Ridker PM, Zipes DP, Shah PK, Willerson JT: From vulnerable plaque to vulnerable patient: a call for new definitions and risk assessment strategies. Part 1. Circulation 2003;108:1664-1672.

10 Finn AV, Nakano M, Narula J, Kolodgie FD, Virmani R: Concept of vulnerable/unstable plaque. Arterioscler Thromb Vasc Biol 2010;30:1282-1292.

-11 Vancraeynest D, Pasquet A, Roelants V, Gerber BL, Vanoverschelde JL: Imaging the vulnerable plaque. J Am Coll Cardiol 2011;57:1961-1979.

12 Markus HS, King A, Shipley M, Topakian R, Cullinane M, Reihill S, Bornstein NM, Schaafsma A: Asymptomatic embolisation for prediction of stroke in the Asymptomatic Carotid Emboli Study (ACES): a prospective observational study. Lancet Neurol 2010;9:663-671.

13 Topakian R, King A, Kwon SU, Schaafsma A, Shipley M, Markus HS, ACES Investigators: Ultrasonic plaque echolucency and emboli signals predict stroke in asymptomatic carotid stenosis. Neurology 2011;77:751-758.

14 Meairs S, Hennerici M: Four-dimensional ultrasonographic characterization of plaque surface motion in patients with symptomatic and asymptomatic carotid artery stenosis. Stroke 1999;30:1807-1813.

15 Bang J, Dahl T, Bruinsma A, Kaspersen JH, Nagelhus Hernes TA, Myhre HO: A new method for analysis of motion of carotid plaques from RF ultrasound images. Ultrasound Med Biol 2003;29:967-976.

-16 Stoitsis J, Golemati S, Nikita KS, Nicolaides AN: Characterization of carotid atherosclerosis based on motion and texture features and clustering using fuzzy c-means. Conf Proc IEEE Eng Med Biol Soc 2004;2:1407-1410.

17 Lenzi GL, Vicenzini E: The ruler is dead: an analysis of carotid plaque motion. Cerebrovasc Dis 2007;23:121-125.

-18 Staub D, Schinkel AF, Coll B, Coli S, van der Steen AF, Reed JD, Krueger C, Thomenius KE, Adam D, Sijbrands EJ, ten Cate FJ, Feinstein SB: Contrast-enhanced ultrasound imaging of the vasa vasorum: from early atherosclerosis to the identification of unstable plaques. JACC Cardiovasc Imaging 2010;3:761-771.

19 Vicenzini E, Giannoni MF, Benedetti-Valentini F, Lenzi GL: maging of carotid plaque angiogenesis. Cerebrovasc Dis 2009;27(suppl 2):48-54.

20 Dong L, Kerwin WS, Chen H, Chu B, Underhill HR, Neradilek MB, Hatsukami TS, Yuan C, Zhao XQ: Carotid artery atherosclerosis: effect of intensive lipid therapy on the vasa vasorum - evaluation by using dynamic contrast-enhanced MR imaging. Radiology 2011;260:224-231.

-21 Saam T, Hatsukami TS, Takaya N, Chu B, Underhill H, Kerwin WS, Cai J, Ferguson MS, Yuan C: The vulnerable, or high-risk, atherosclerotic plaque: noninvasive MR imaging for characterization and assessment. Radiology 2007;244: $64-77$.

22 Markl M, Dudler P, Fydrychowicz A, Strecker C, Weiller C, Hennig J, Harloff A: Optimized 3D bright blood MRI of aortic plaque at 3 T. Magn Reson Imaging 2008;26:330-336. 
23 Saam T, Underhill HR, Chu B, Takaya N, Cai J, Polissar NL, Yuan C, Hatsukami TS: Prevalence of American Heart Association type VI carotid atherosclerotic lesions identified by magnetic resonance imaging for different levels of stenosis as measured by duplex ultrasound. J Am Coll Cardiol 2008;51:1014-1021.

24 Harloff A, Albrecht F, Spreer J, Stalder AF, Bock J, Frydrychowicz A, Schöllhorn J, Hetzel A, Schumacher M, Hennig J, Markl M: 3D blood flow characteristics in the carotid artery bifurcation assessed by flow-sensitive 4D MRI at 3T. Magn Reson Med 2009;61:65-74.

25 Davies PF: Flow-mediated endothelial mechanotransduction. Physiol Rev 1995;75:519-560.

26 Chiu JJ, Chien S: Effects of disturbed flow on vascular endothelium: pathophysiological basis and clinical perspectives. Physiol Rev 2011;91:327-387.

27 Cheng C, Tempel D, van Haperen R, van der Baan A, Grosveld F, Daemen MJ, Krams R, de Crom R: Atherosclerotic lesion size and vulnerability are determined by patterns of fluid shear stress. Circulation 2006;113:2744-2753.

28 Lee SW, Antiga L, Spence JD, Steinman DA: Geometry of the carotid bifurcation predicts its exposure to disturbed flow. Stroke 2008;39:2341-2347.

29 Markl M, Wegent F, Zech T, Bauer S, Strecker C, Schumacher M, Weiller C, Hennig J, Harloff A: In vivo wall shear stress distribution in the carotid artery: effect of bifurcation geometry, internal carotid artery stenosis, and recanalization therapy. Circ Cardiovasc Imaging 2010;3:647-655.

-30 Yang C, Canton G, Yuan C, Ferguson M, Hatsukami TS, Tang D: Advanced human carotid plaque progression correlates positively with flow shear stress using follow-up scan data: an in vivo MRI multi-patient 3D FSI study. J Biomech 2010;43:2530-2538.

-31 Slager CJ, Wentzel JJ, Gijsen FJ, Thury A, van der Wal AC, Schaar JA, Serruys PW: The role of shear stress in the destabilization of vulnerable plaques and related therapeutic implications. Nat Clin Pract Cardiovasc Med 2005;2:456464.

-32 Gao H, Long Q, Kumar Das S, Halls J, Graves M, Gillard JH, Li ZY: Study of carotid arterial plaque stress for symptomatic and asymptomatic patients. J Biomech 2011;44:2551-2557. 